\title{
EL CONOCIMIENTO EN ACCESO ABIERTO EN LOS PLANES DE ACCION TUTORIAL DE LAS UNIVERSIDADES ESPAÑOLAS
}

\author{
Alejandra Hernández-Ruiz ${ }^{1}$ : Universidad de Alicante. España \\ alejandra.hernandez@ua.es
}

Conchi Campillo-Alhama: Universidad de Alicante. España concepcion.campillo@ua.es

José Daniel Álvarez-Teruel: Universidad de Alicante. España josedaniel.alvarez@ua.es

\section{RESUMEN}

El Espacio Europeo de Educación Superior revitaliza la competencia tutorial como uno de los ejes nucleares sobre los que debe sustentarse la actividad docente. Para satisfacer y cumplir los derechos y los deberes de alumnos y profesores, en materia de acción tutorial, el sistema educativo español planifica las actividades de orientación y asesoramiento al estudiante en los Planes de Acción Tutorial (PAT). En este trabajo nos proponemos, como objetivo general, elaborar una guía de los recursos educativos de acción tutorial que las universidades españolas difunden en acceso abierto y/o en sus páginas web institucionales, para facilitar la orientación del alumnado y el trabajo del tutor. La realización de este estudio podría constituir la piedra angular de la creación de futuras redes de colaboración docente interuniversitarias en acción tutorial que potencien la apertura del profesorado hacia otros entornos educativos.

PALABRAS CLAVE: Conocimiento abierto -Acción Tutorial - Universidades España - Recursos educativos

\footnotetext{
${ }^{1}$ Autor correspondiente

Alejandra Hernández-Ruiz: Profesora Ayudante Doctora. Universidad de Alicante. España Correo: alejandra.hernandez@ua.es
} 


\title{
OPEN ACCESS TO KNOWLEDGE IN TUTORIAL ACTION PLANS AT SPANISH UNIVERSITIES
}

\begin{abstract}
The European Higher Education Area revitalizes tutorial competence as one of the core elements which should underlie the teaching profession. To meet and fulfill the rights and duties of students and teachers, in terms of tutoring, the Spanish educational system plans the activities of guidance and counseling students in the Tutorial Action Plans. In this paper we propose, as a general objective, to develop an educational resource guide in tutorial action that Spanish universities disseminate in open access and / or in institutional web pages, to help guide the students and the tutor's work. The completion of this study could form the cornerstone of future development of inter-university teaching collaboration networks in tutorial action to enhance the openness of teachers to other educational settings.
\end{abstract}

KEY WORDS: Open Knowledge - Tutorial Action - Universities - Spain Educational Resources

\section{INTRODUCCIÓN}

\subsection{El paradigma del conocimiento en acceso abierto}

Para comprender la filosofía del conocimiento abierto, tal y como apuntan Labastida e Iglesias (2006), es necesario mencionar las tres declaraciones que constituyen el eje fundacional de este movimiento.

En primer lugar, la Declaración de Budapest (la denominada "Budapest Open Access Initiative") fue promulgada en febrero de 2002 como resultado de un encuentro convocado en esta ciudad por el "Open Society Institute" (OSI) en diciembre de 2001. Básicamente, el propósito de esta reunión fue acelerar el progreso del esfuerzo internacional en la libre difusión en Internet de la producción científica de todos los campos de conocimiento.

En segundo lugar, un año más tarde, el 11 de abril de 2003, en Maryland, tuvo lugar un encuentro para estimular la discusión, entre la comunidad científica del área de biomedicina, sobre cómo actuar para conseguir el objetivo global de difundir, en abierto, la literatura científica. Las conclusiones de esta reunión dieron lugar a la Declaración de Bethesda ("Bethesda Statement on Open Access Publishing") que incluye una definición de la publicación en acceso abierto. Así, una obra de estas características debe cumplir las siguientes condiciones: 
a) El autor/los autores y el titular/los titulares de los derechos de autor concede/conceden a todos los usuarios un derecho libre, irrevocable, universal y perpetuo de acceso y licencia para copiar, usar, distribuir, transmitir y presentar el trabajo públicamente, así como hacer y distribuir obras derivadas, en soporte digital para un uso responsable, sujeto al reconocimiento apropiado de la autoría, así como al derecho a hacer un número limitado de copias impresas para uso personal.

b) Una versión completa de la obra y todos los materiales adicionales, incluida una copia de los permisos citados anteriormente, en un formato electrónico estándar apropiado, se depositará de forma inmediata a la publicación inicial en, al menos, un repositorio en línea apoyado por una institución académica, una sociedad académica, una agencia gubernamental o cualquier otra organización debidamente establecida que persiga facilitar el acceso abierto, la distribución sin restricciones, la interoperabilidad y el archivo a largo plazo.

Y, en tercer lugar, cuatro meses más tarde, en octubre de 2003, Berlín acoge una conferencia sobre el acceso abierto al conocimiento en Ciencias y Humanidades. Como resultado de este encuentro se promulgó la Declaración de Berlín que constituyó un hito histórico para este movimiento (Hedlund \& Rabow, 2007).

De forma sucinta, las instituciones firmantes de esta declaración tienen la obligación de asumir Internet como medio para la distribución de conocimiento. Hasta la fecha, 324 instituciones $^{2}$ se han adherido a la Declaración de Berlín, de entre las cuales, 22 universidades españolas han suscrito este compromiso (ver tabla I).

Tabla 1. Universidades españolas firmantes de la Declaración de Berlín

\begin{tabular}{|c|c|}
\hline UNIVERSIDAD & FECHA DE ADHESIÓN \\
\hline Universidad de Murcia & 18.05 .2011 \\
\hline Universidad Politécnica de Cartagena & 01.12 .2010 \\
\hline Universidad de Granada & 12.11 .2010 \\
\hline Universidad de Huelva & 08.07 .2010 \\
\hline Universitat de Girona & 26.04 .2010 \\
\hline Universitat de Barcelona & 11.12 .2009 \\
\hline Universidad de Salamanca & 27.02 .2009 \\
\hline
\end{tabular}

\footnotetext{
${ }^{2}$ Véase el listado de organizaciones signatarias en http://webcache.googleusercontent.com/search?q=cache:fgYX3rddSgUJ:oa.mpg.de/lang/en-
} 


\begin{tabular}{|c|c|}
\hline Universitat de València & 30.09 .2008 \\
\hline Universitat d'Alacant, Universidad de Alicante & 09.11 .2006 \\
\hline Universitat Pompeu Fabra & 12.07 .2006 \\
\hline
\end{tabular}

\begin{tabular}{|c|c|}
\hline Universidad de Alcalá de Henares & 12.06 .2006 \\
\hline Universidad Autónoma de Madrid & 12.06 .2006 \\
\hline Universidad Carlos III de Madrid & 12.06 .2006 \\
\hline Universidad Politécnica de Madrid & 12.06 .2006 \\
\hline Universidad Rey Juan Carlos & 12.06 .2006 \\
\hline Universidad Nacional de Educación a Distancia & 12.06 .2006 \\
\hline Universitat Oberta de Catalunya & 02.06 .2006 \\
\hline & 08.03 .2006 \\
\hline Universitat de Vic & 10.02 .2006 \\
\hline Universitat de Lleida & 26.01 .2006 \\
\hline Universidade da Coruña & 10.10 .2005 \\
\hline Universitat Jaume I & 12.06 .2006 \\
\hline Universidad Complutense de Madrid & \\
\hline
\end{tabular}

Fuente: elaboración propia

En la revisión de la literatura sobre el paradigma del conocimiento abierto existen diversos estudios que analizan las políticas de promoción de este movimiento en diversas universidades españolas (Casals, 2010); (Llorens, Bayona, Gómez \& Sanguino, 2010); (Lloret, Calatrava \& Torres, 2010; (Merlo \& Ferreras, 2010). Sin embargo, no hay ninguna referencia previa que focalice su atención sobre la adopción de los fundamentos de este movimiento como medio para la divulgación de los materiales docentes de los planes de acción tutorial de las instituciones españolas de educación superior. 


\subsection{La regulación de la acción tutorial en la normativa española}

Desde los años 70, con la ley 14/1970, de 4 de agosto, General de Educación y Financiamiento de la Reforma Educativa, la normativa española contempla la orientación educativa y profesional para la elección de estudios y actividades laborales como un derecho básico de los estudiantes en todas las etapas educativas (art. 125.2).

En un contexto institucional que aspira a democratizar la educación mediante el establecimiento de una etapa de formación general básica y gratuita para todos los ciudadanos, los servicios de orientación se conciben como necesarios para conseguir un ajuste entre las capacidades, aptitudes y vocaciones de los estudiantes y la elección consciente y responsable de opciones académicas y laborales (art.9.4).

Para la primera de las tareas de orientación, la educativa, este texto legal establece la obligatoriedad de establecer un régimen de tutorías desde la incorporación del estudiante a los centros educativos (art. 127.1). Para la segunda, la orientación profesional, la ley tan sólo habla de proporcionar al alumnado información relacionada con la situación y perspectivas de empleo (art.127.2).

Sin embargo, en el ámbito universitario, el apoyo legislativo a la acción tutorial y a la figura del profesor tutor se limitó, como señala Sáenz de Miera (Sáenz de Miera, 2001), a cuestiones vinculadas con la actividad académica del discente, sin considerar temas personales y profesiones del alumnado.

Habrá que esperar a la década de los 90, con la Ley Orgánica 1/1990, de 3 de octubre, de Ordenación General del Sistema Educativo (LOGSE), para asistir a una revalorización de la acción tutorial (Del Valle, 2004). Así, bajo esta reforma educativa, la atención psicopedagógica y la orientación educativa y profesional se configura como uno de los ejes sobre los que se debe asentar la actividad educativa, junto con otros aspectos como la formación integral del alumnado y el desarrollo de las capacidades creativas y del espíritu creativo (art.3).

Además, se concibe la orientación educativa y profesional como uno de los factores que favorecen la calidad y la mejora de la enseñanza, al mismo nivel que otros asuntos como la cualificación y formación del profesorado y la innovación y la investigación educativa, entre otros (art. 55). En consecuencia, este texto legal incluye, por primera vez, como función docente, la tutoría y la orientación de los alumnos (art.60).

En esta misma línea, la vigente ley educativa española, la Ley Orgánica 2/2006, de 3 de mayo, de Educación, reconoce como un derecho básico de los estudiantes "la orientación educativa y profesional como medio para el logro de una formación personalizada que propicie una educación integral en conocimientos, destrezas y valores" (art.1.f). De este modo, debe ser tarea del docente la orientación educativa, académica v profesional de los alumnos (art. 3.d). 
En el ámbito universitario, la Ley Orgánica 6/2001, de 21 de diciembre, de Universidades, recoge el asesoramiento y asistencia por parte de profesores y tutores entre los derechos de los discentes (art. 2.e). Sin embargo, no hay ninguna mención específica al rol del profesor tutor.

Años más tarde, la Ley Orgánica 4/2007, de 12 de abril, por la que se modifica la LOU 2001, encomienda al Gobierno la aprobación del Estatuto del Estudiante Universitario (disposición adicional decimocuarta). Así, en la redacción del Real Decreto 1791/2010, de 30 de diciembre, por el que se aprueba el Estatuto del Estudiante Universitario, se incluye, como un derecho común de los estudiantes universitarios, en particular, del estudiante pre-universitario, "la promoción por parte de las universidades de programas de información y orientación a sus futuros estudiantes que favorezcan la transición activa a la universidad" (art. 7.1.a).

Por lo que se refiere a los estudiantes universitarios de grado, se establece como derecho "recibir orientación y tutoría personalizadas en el primer año y durante los estudios, para facilitar la adaptación al entorno universitario y el rendimiento académico, así como en la fase final con la finalidad de facilitar la incorporación laboral, el desarrollo profesional y la continuidad de su formación universitaria" (art. 8.1.f).

El Estatuto del Estudiante Universitario también prevé una serie de derechos específicos para los estudiantes de Máster, entre los que se encuentra el de "recibir orientación y tutoría personalizadas para facilitar el rendimiento académico, la preparación para la actividad profesional o la iniciación a la investigación" (art. 9.e).

La importancia de la acción tutorial en el Espacio Europeo de Educación Superior queda patente en el capítulo V del Estatuto del Estudiante Universitario. Para satisfacer el derecho de los estudiantes a recibir orientación y seguimiento de carácter transversal sobre su titulación (art. 19.1), el Estatuto prevé la figura del coordinador y tutor de titulación, cuya misión será llevar a cabo una "orientación de calidad, dirigida a reforzar y complementar la docencia como formación integral y crítica de los estudiantes y como preparación para el ejercicio de actividades profesionales" (art.19.2).

En particular, en el art.20.2, se identifican los objetivos que se persiguen con la acción tutorial que se inicia con la transición del estudiante al entorno universitario y finaliza con el desarrollo inicial de la carrera profesional.

De forma paralela al Estatuto del Estudiante Universitario, la Ley Orgánica 4/2007, de 12 de abril, en su disposición adicional sexta, delega en el Gobierno la aprobación, mediante Real Decreto, del estatuto del personal docente o investigador universitario (disposición adicional sexta). En lo que acción tutorial se refiere, el Borrador del 
Estatuto del Personal Docente e Investigador incluye, entre las actividades docentes del PDI, la tutoría, orientación y atención a los estudiantes, individuales y grupales.

Sin embargo, a la espera de la aprobación definitiva del Estatuto del PDI, el borrador adolece de una falta de correspondencia entre los derechos de los estudiantes, plasmados en el Estatuto del Estudiante Universitario y los deberes del cuerpo docente en materia de acción tutorial.

\subsection{Los planes de acción tutorial en el sistema universitario español}

La educación contribuye a la formación del individuo en tres niveles: en su aspecto laboral o profesional, en su papel como ciudadano o miembro de una polis, y en la formación integral del individuo como ser humano (Sicinski, A., 1990, p. 234).

Estos tres niveles son sintetizados por García (2008) en dos facetas: (1) La faceta instructiva entendida como la dimensión cognoscitiva del aprendizaje y (2) la faceta formativa, concebida como el desarrollo y transformación de actitudes, valores, hábitos, comportamientos, etc.

En el contexto actual de incorporación de las universidades españolas al Espacio Europeo de Educación Superior, la función tutorial se presenta como una herramienta indispensable para ayudar al desarrollo personal, académico y profesional del alumnado (Sobrado, 2008) y, desde una perspectiva conciliadora, como punto de unión entre la orientación académica y profesional del discente (García, 2008).

La reforma de la convergencia europea supone, como subraya García (2008), la adopción de cambios en diversos órdenes: en las titulaciones, en los actuales curricula universitarios, en los objetivos de la enseñanza superior, en las metodologías y en los roles de los protagonistas del proceso de enseñanza- aprendizaje.

En este contexto, el rol del docente universitario evoluciona desde la figura de un transmisor de conocimientos a su función como facilitador, guía, orientador, tutor y asesor del estudiante, además de investigador crítico y gestor (Gairín, Feixas, Guillamón \& Quinquer, 2004); (Lázaro, 2008); (López \& Sola, 2003).

En este marco de trabajo, el sistema educativo español planifica las actividades de orientación y asesoramiento al estudiante en los Planes de Acción Tutorial (PAT). Como apuntan Álvarez, Lorca y García (Álvarez, Lorca \& García, 2010), Jiménez (Jiménez, 2010) y Gairín (Gairín et al., 2004), la idea de adoptar Planes de Acción Tutorial como apoyo al estudiante en su faceta académica y profesional no es algo nuevo, pues ya el Informe Bricall (Bricall, 2000) recomendaba que las instituciones de enseñanza superior establecieran los servicios de asesoramiento y apoyo al estudiante como una parte central de sus prestaciones. 
Según la doble clasificación de las tutorías en el ámbito universitario que proponen Mateos y Montanero (2008), estos planes formarían parte de las tutorías vinculadas a una titulación. En este sentido, el Programa o Plan de Acción Tutorial puede ser entendido como un documento organizativo que sirve para vertebrar las diferentes acciones en materia de acción tutoría capaces de favorecer la excelencia académica, la formación integral y la atención a todos los estudiantes (Pantoja \& Campoy, 2009).

En la literatura sobre los Programas o Planes de Acción Tutorial el enfoque actualmente dominante, en la Orientación Universitaria, es la educación para la carrera, de modo que la orientación debe abarcar todos los ámbitos y momentos de la carrera personal y universitaria del estudiante: entrada a la universidad, permanencia y la transición al mundo laboral) (Gairín et al., 2004:62).

En función de estas tres etapas, los Programas de Acción Tutorial deben estar dirigidos a la consecución de tres objetivos: (1) la integración formativa plena de los estudiantes, (2) el desarrollo de metodologías y estrategias de estudio y aprendizaje, y (3) la inserción laboral de los estudiantes (Sobrado, 2008).

En esta línea, Cano (2008) propone trabajar la Acción Tutorial desde una triple perspectiva: (1) Tutoría Vocacional (tránsito Bachillerato-Universidad), (2) Tutoría Académica-Universitaria (docente y orientadora) y (3) Tutoría Profesional-Laboral (inserción laboral).

De forma más específica, y como señala Martínez (2011), el PAT debería abarcar actuaciones dirigidas a facilitar la integración del estudiante en su entorno más inmediato (institución y titulación), a guiar el desarrollo de las competencias transversales y específicas, a fomentar la iniciativa y el auto-conocimiento por parte del alumno, a informar y orientar sobre salidas profesionales, a favorecer la transición a la vida activa, etc.

Ante el respaldo institucional a la acción tutorial a través del Real Decreto 1393/2007, por el que se establece la ordenación de las enseñanzas universitarias oficiales, las universidades españolas deben apostar por implementar Planes de Acción Tutorial como uno de los criterios de calidad que permitirá la verificación de sus titulaciones oficiales (artículos 4.1 y 4.3 anexo I). 


\section{METODOLOGÍA}

\subsection{El estudio: guía de materiales docentes de acción tutorial}

\section{Objetivos}

En este contexto, en este trabajo nos proponemos, como objetivo general, elaborar una guía de los recursos educativos de acción tutorial que las universidades españolas difunden en acceso abierto y/o en sus páginas web institucionales, para facilitar la orientación del alumnado y el trabajo del tutor en todos los ámbitos y momentos de la carrera personal y universitaria del estudiante (entrada a la universidad, permanencia y transición al mundo laboral).

Dado que nuestro interés reside en compilar los recursos disponibles de forma libre y gratuita en Internet que ofrecen las instituciones españolas de educación superior, centramos nuestra atención en observar, en primer lugar, la visibilidad web de los planes de acción tutorial. Para ello, analizamos la información proporcionada en los sitios $w^{3} b^{3}$ de las universidades españolas que ofrecen sistemas de información, apoyo y asesoramiento a sus estudiantes, de forma institucionalizada y consensuada, a través de un Programa o Plan de Acción Tutorial.

Para nuestra investigación adoptamos la siguiente secuencia de búsqueda:

a) Localización en la página principal de algún enlace directo de acción tutorial.

b) Búsqueda por centros: localización de enlace directo y búsqueda en los enlaces de alumnado, docencia y calidad.

c) Búsqueda en el motor de búsqueda del sitio web.

Como fuente de investigación secundaria, para poder localizar con mayor precisión los centros y universidades en los que existen programas de acción tutorial, orientamos nuestra búsqueda con la revisión de la literatura académica sobre convergencia europea y función tutorial.

\section{ANÁLISIS Y DISCUSIÓN}

De las 74 universidades españolas objeto de nuestro estudio, 41 centros (el 55,4\%) tiene planes de acción tutorial de forma visible en sus webs institucionales $\mathrm{y}$, entre éstos, 18 instituciones (el 43,9\%) ponen a disposición de la comunidad universitaria recursos educativos para la orientación y el asesoramiento del alumnado.

\footnotetext{
${ }^{3}$ Los sitios web de las 74 universidades españolas fueron visitados y consultados durante el mes de julio
} 
Sin embargo, a pesar de la disposición libre y gratuita en Internet de diversos materiales docentes, sólo una de las instituciones objeto de estudio cumple con los dos condiciones que debe satisfacer una publicación en acceso abierto. Así, la Universidad de Murcia deposita en su repositorio institucional (Digitum), con una licencia creative commons, su guía sobre "Las orientaciones para el desarrollo de la acción tutorial en la Facultad de Biología". En el resto de las universidades consideradas es inexistente el auto-archivo del recurso en un repositorio institucional, así como la gestión de los derechos de autor.

Los materiales seleccionados se agruparon en función de su público objetivo: profesorado tutor o alumnado. Para nuestro estudio seleccionamos aquellos recursos que pueden resultar útiles a los miembros de otros centros universitarios. Así, materiales con contenido de carácter localista, como los recursos para conocer mejor el propio centro universitario, no forman parte de nuestra base documental.

En la tabla I se muestra la relación de recursos web de apoyo a la labor del tutor que ofrecen las universidades españolas.

Tabla 2. Materiales de acción tutorial para el tutor

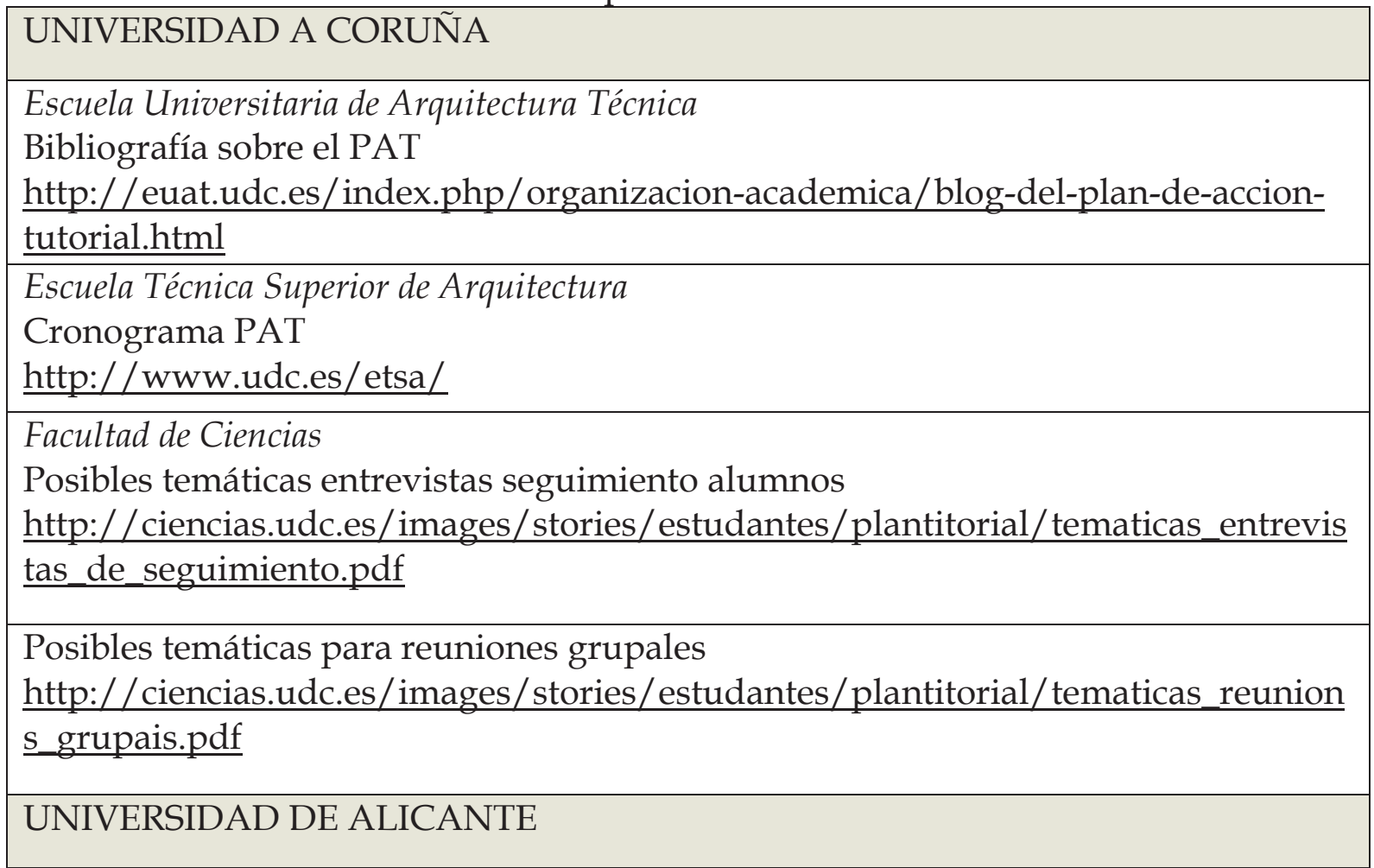


La acción tutorial en el bachillerato y su proyección en la Universidad: recursos personales http://web.ua.es/es/ice/documentos/tutorial/material/taller-iii.pdf http:// web.ua.es/es/ice/documentos/tutorial/material/taller-pat-3.pdf

Orientar para poner en valor al alumno como futuro profesional http://web.ua.es/es/ice/documentos/tutorial/material/orientar-para-poner-envalor.pdf

Material I Jornada de trabajo sobre la acción tutorial universitaria 


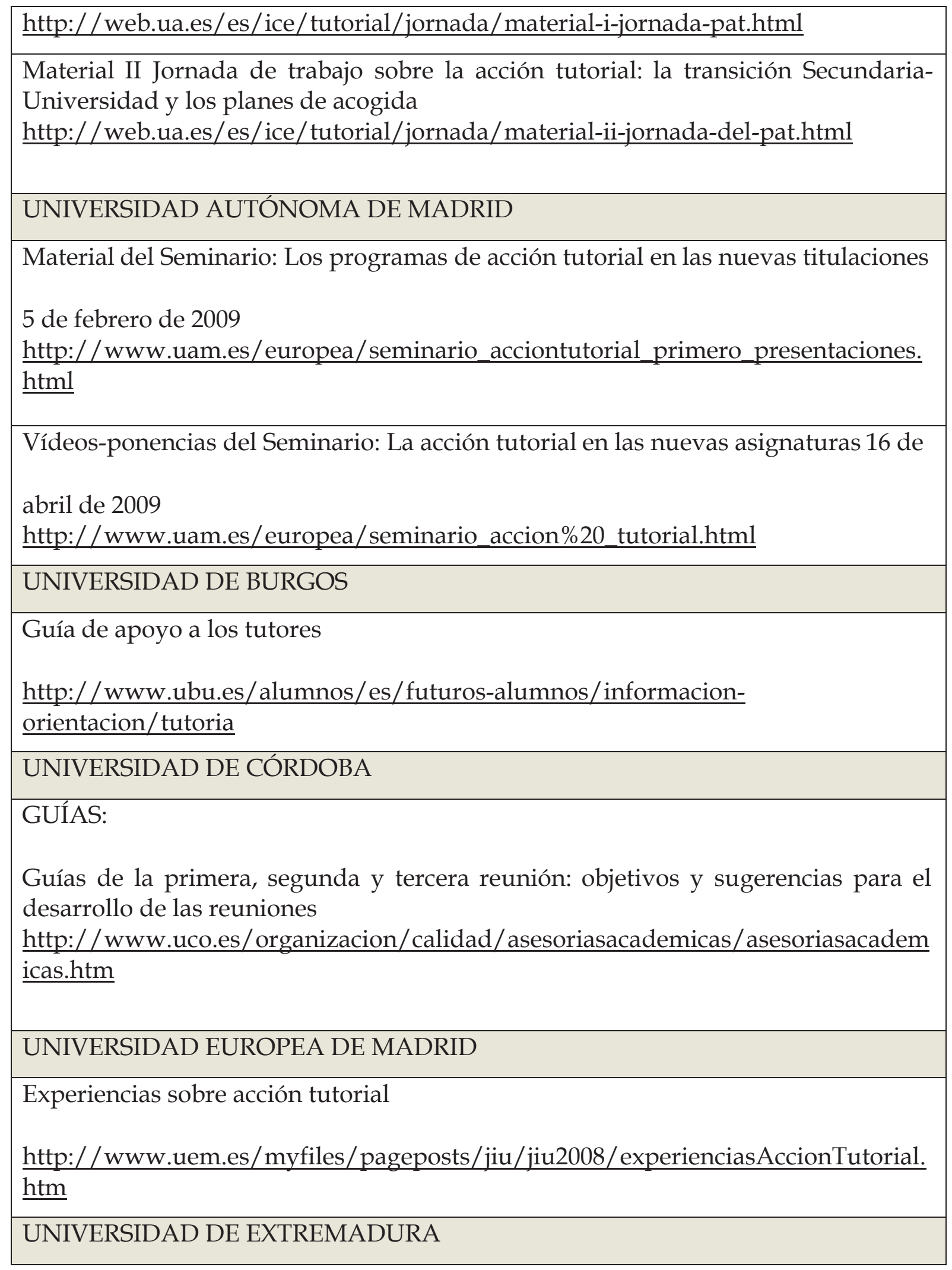




\section{Facultad de Veterinaria}

Sugerencias sobre temas a tratar en las reuniones http:/ / www.unex.es/conoce-lauex/estructuraacademica/centros/veterinaria/contenido_portlets_configurables/car_20090327_0 01/car_20090528_001

\section{UNIVERSITAT DE GIRONA}

Actividad para el desarrollo del apoyo emocional

http://www.udg.edu/LinkClick.aspx?fileticket=TbCK0ybYxns\%3d\&tabid=13174

0 1

Actividad para el desarrollo del apoyo emocional: escuchar sin juzgar

http:/ / www.udg.edu/LinkClick.aspx?fileticket=0Ihkau6EPAI\%3d\&tabid=13174\&

1 Amomomon $\mathrm{rC}$

Actividad para el desarrollo del reconocimiento

http:/ / www.udg.edu/LinkClick.aspx?fileticket=4iEob31XxMI\%3d\&tabid=13174\&1 $\underline{\text { anguage }=\text { ca-ESc }}$

\section{UNIVERSIDAD DE LA LAGUNA}

Modelo orientación tutoría

http://www.ull.es/Private/folder/institucional/ull/calidad/Alicia/ModeloOrie ntacionTutoria/DocumentosGenerales/ModeloOrientacionTutoriaULL.pdf

Comunicaciones de las I Jornadas de Acción Tutorial de la ULL, abril 2011

http://www.ull.es/view/institucional/ull/Accion_tutorial/es UNIVERSITAT DE LLEIDA

Estilos de autoaprendizaje y planificación

http:// www.ice.udl.cat/uou/patudl/activitatmoment2.pdf

Comportamiento asertivo y reestructuración cognitiva

http://www.ice.udl.cat/uou/patudl/activitatmoment3.pdf

Competencias personales: El plan de estudios

Competencias personales: Las competencias profesionales y nuestros estudios 


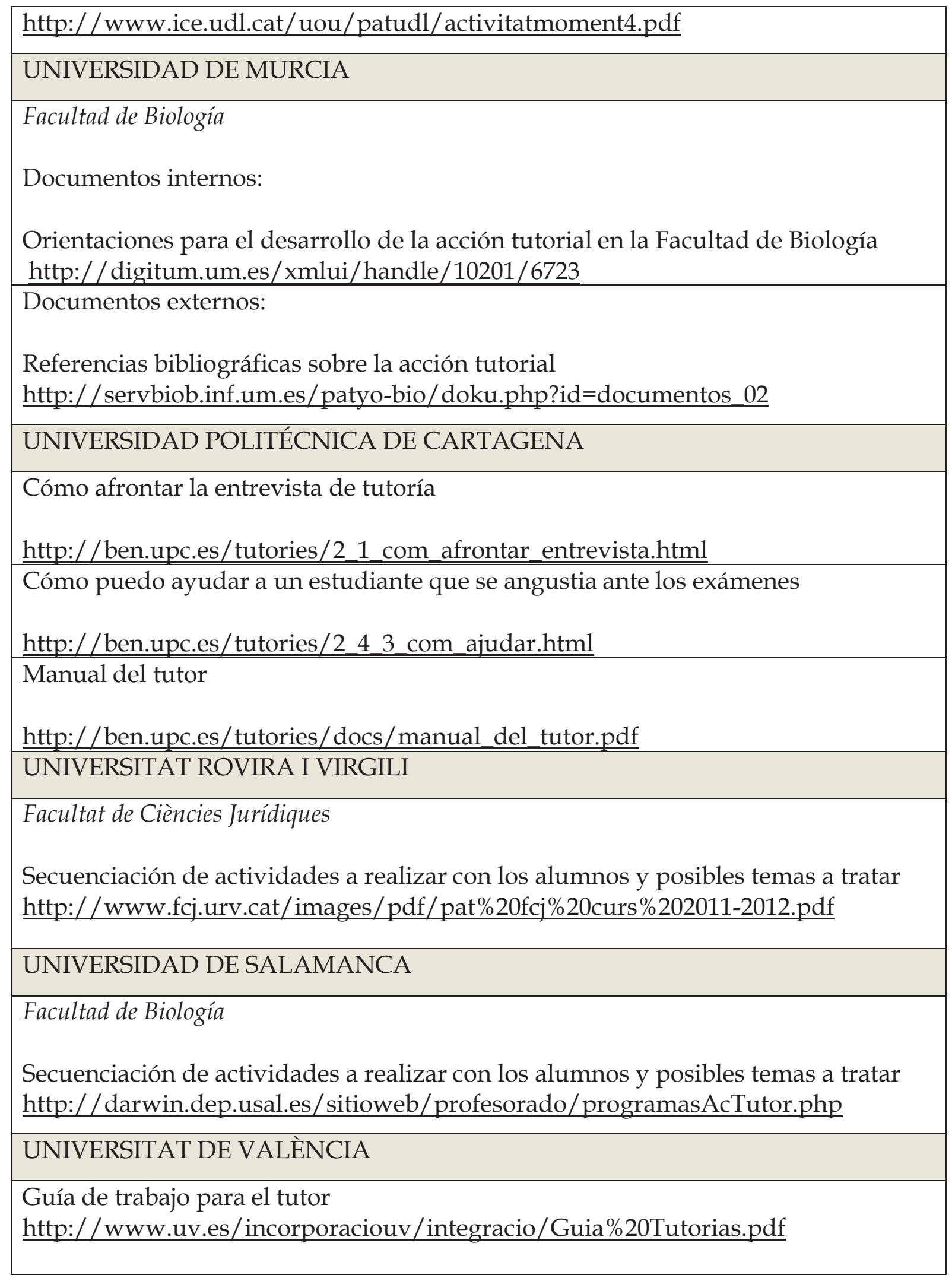




\begin{tabular}{|l|}
\hline UNIVERSIDAD DE VALLADOLID \\
$\begin{array}{l}\text { Secuenciación de reuniones a realizar con los alumnos y posibles temas a tratar } \\
\text { http://www.uva.es/uva/export/portal/com/bin/contenidos/gobiernoUVA/Vic } \\
\text { errectorados/VicerectoradoCalidadInnovacion/Innovacion_educativa/Convocato } \\
\text { rias/Orienta09/1244463815705_anexo_iii_orienta.doc }\end{array}$ \\
\hline UNIVERSIDAD DE ZARAGOZA \\
$\begin{array}{l}\text { Centro Politécnico Superior } \\
\text { Guía primera reunión }\end{array}$ \\
\hline $\begin{array}{l}\text { Guía segunda reunión } \\
\text { http://wwww.cps.unizar.es/docs/2007/PPAT/7.guia_2_reunion.pdf }\end{array}$ \\
\hline $\begin{array}{l}\text { Guía tercera reunión } \\
\text { http://www.cps.unizar.es/docs/2007/PPAT/10.guia_3_reunion.pdf }\end{array}$ \\
\hline
\end{tabular}

Fuente: elaboración propia

Por su parte, en la tabla II se recogen los materiales destinados a facilitar la orientación del alumnado de forma autónoma.

Tabla 3. Materiales de acción tutorial para el alumnado

\begin{tabular}{|c|}
\hline UNIVERSIDAD DE ALICANTE \\
\hline $\begin{array}{l}\text { Decálogo para la eficacia de la comunicación no verbal en } \\
\text { los procesos de selección de personal } \\
\text { http://web.ua.es/es/ice/documentos/tutorial/material/decalogo-de }\end{array}$ \\
\hline$\underline{\text { comunicacion-no-verbal.pdf }}$ \\
\hline $\begin{array}{l}\text { Instrucciones para utilizar el curriculum vitae } \\
\text { Europass } \\
\text { http://web.ua.es/es/ice/documentos/tutorial/mat } \\
\text { erial/instrucciones-para- cv.pdf }\end{array}$ \\
\hline
\end{tabular}




\begin{tabular}{|c|}
\hline UNIVERSIDAD CATÓLICA DE MURCIA \\
\hline $\begin{array}{l}\text { Organización y planificación } \\
\text { http:// www.ucam.edu/servicios/tutorias/orientacion-del-cuerpo-de- } \\
\text { tutores/organizacion-y-planificacion }\end{array}$ \\
\hline $\begin{array}{l}\text { Técnicas de estudio en la Universidad } \\
\text { http:/ / www.ucam.edu/servicios/tutorias/orientacion-del-cuerpo-de- } \\
\underline{\text { tutores/tecnicas-de-estudio-en-la-universidad }}\end{array}$ \\
\hline $\begin{array}{l}\text { Cómo abordar los exámenes } \\
\text { http://www.ucam.edu/servicios/tutorias/orientacion-del-cuerpo-de- } \\
\text { tutores/bfcomo-abordar-los-examenes }\end{array}$ \\
\hline $\begin{array}{l}\text { Cuestiones básicas de vital importancia } \\
\text { http://www.ucam.edu/servicios/tutorias/orientacion-del-cuerpo-de- } \\
\underline{\text { tutores/cuestiones-basicas-de-vital-importancia }}\end{array}$ \\
\hline $\begin{array}{l}\text { Aspecto relacional. Habilidades sociales } \\
\text { http://www.ucam.edu/servicios/tutorias/orientacion-del-cuerpo-de- } \\
\text { tutores/aspecto-relacional.-habilidades-sociales }\end{array}$ \\
\hline \multirow{2}{*}{$\begin{array}{l}\text { Adicciones } \\
\underline{\text { http://www.ucam.edu/servicios/tutorias/orientacion-del-cuerpo-de- }} \\
\underline{\text { tutores/adicciones }}\end{array}$} \\
\hline \\
\hline \multirow{2}{*}{$\begin{array}{l}\text { Educación para el ocio y el tiempo libre } \\
\text { http://www.ucam.edu/servicios/tutorias/orientacion-del-cuerpo-de- } \\
\underline{\text { tutores/educacion-para-el-ocio-y-el-tiempo-libre }}\end{array}$} \\
\hline \\
\hline \multirow{2}{*}{$\begin{array}{l}\text { Valores } \\
\text { http:// www.ucam.edu/servicios/tutorias/orientacion-del-cuerpo-de- } \\
\underline{\text { tutores/valores }}\end{array}$} \\
\hline \\
\hline UNIVERSIDAD DE LA LAGUNA \\
\hline Facultad de CC. Económicas y Empresariales \\
\hline Técnicas de estudio y organización del trabajo académico \\
\hline
\end{tabular}


http://www.ull.es/Private/folder/centros/fceye/2010/Tecnicas_estudio_POAT_

FCEYE.pdf

UNIVERSITAT DE LLEIDA

Actividades de autoformación Estilos de

autoaprendizaje

http://www.ice.udl.cat/uou/patudl/2/

Comunicación Asertiva

http://www.ice.udl.cat/uou/patudl/3/

Autoestima

http://www.ice.udl.cat/uou/patudl/3a/

Fuente: elaboración propia

Como se puede observar, las universidades que utilizan Internet como medio para divulgar sus recursos educativos de acción tutorial son, en su mayoría, instituciones de carácter público. Así, de los dieciocho centros, sólo dos (Universidad Europea de Madrid y Universidad Católica de San Antonio de Murcia) son de titularidad privada.

Por otro lado, la mayoría de los recursos encontrados están destinados a orientar y apoyar la labor del profesor tutor. Entre estos materiales podemos encontrar:

Documentos de trabajo para el tutor:

a) Cronograma PAT.

b) Cómo afrontar la entrevista de tutoría y cómo ayudar a los estudiantes.

c) Guías de las reuniones y posibles temáticas a tratar en las entrevistas.

d) Material de Jornadas de trabajo sobre la acción tutorial universitaria en la UA, la UEM y la ULL.

Documentos orientados a la formación de los tutores:

a) Bibliografía PAT.

b) La acción tutorial en el bachillerato y su proyección en la

c) Universidad: recursos personales.

d) Orientar para poner en valor al alumno como futuro profesional.

Documentos dirigidos a la formación de los estudiantes:

a) Actividad para el desarrollo del apoyo emocional y el reconocimiento

b) Estilos de autoaprendizaje y planificación.

c) Competencias personales.

d) Comportamiento asertivo y reestructuración cognitiva. 
Por su parte, los materiales formativos dirigidos al alumnado versan sobre:

Orientación personal

a) Cuestiones básicas de vital importancia.

b) Adicciones.

c) Aspecto relacional. Habilidades sociales.

d) Educación para el ocio y el tiempo libre.

e) Valores.

Orientación académica

a) Estilos de autoaprendizaje.

b) Técnicas de estudio para universitarios.

c) Organización y planificación.

d) Cómo abordar los exámenes.

Orientación profesional

a) Decálogo para la eficacia de la comunicación no verbal en los procesos de selección de personal.

b) Instrucciones para utilizar el curriculum vitae Europass.

c) Comunicación asertiva.

\section{CONCLUSIONES}

Esta investigación constituye el primer estudio realizado en nuestro país sobre la adopción del paradigma del conocimiento en acceso abierto para la divulgación de recursos educativos de acción tutorial.

Pese a que la acción tutorial es un elemento nuclear de la calidad de los sistemas de educación superior, tal y como hemos podido apreciar en nuestro estudio, son pocas las instituciones españolas que incluyen en sus páginas web sus Programas o Planes de acción tutorial.

Dado que, como señalan Carrillo y Castillo (2010), la sede web de las universidades constituye un vehículo útil de comunicación para sus marcas, las instituciones españolas deberían fomentar: (1) la visibilidad web de sus programas de acción tutorial y (2) la adopción de políticas de promoción de acceso libre al conocimiento, de forma específica, en sus planes de orientación.

Así, junto a otros indicadores de calidad de la institución educativa como la investigación y la producción científica o la ratio alumno-profesor, los programas de orientación y atención al alumnado deberían ser uno de los criterios clave para la selección del centro educativo. 
Las dificultades encontradas en nuestro trabajo para localizar los planes de acción tutorial de cada universidad y cada centro aconsejan acometer una serie de estudios que permitan clarificar y homogeneizar la información ofrecida al alumnado. Así, en la línea del trabajo de Tortosa, Moliner, López y López (Tortosa, Moliner, López \& López 2010), sería recomendable estudiar el acceso web del alumnado a los programas de acción tutorial de los distintos centros de una misma institución educativa, para, con estos datos, poder establecer una política institucional que establezca los parámetros a seguir para asegurar la presencia web de los programas de acción tutorial en las universidades españolas.

En este sentido, con el fin de coordinar la actuación de las distintas instituciones de educación superior en materia de acción tutorial, proponemos como punto de partida la creación de una red docente de trabajo interuniversitaria que permita paliar lagunas como la ausencia de un documento marco sobre los derechos y deberes de alumnos y profesores y fomente la apuesta de las universidades por el conocimiento abierto y la libre difusión de recursos que puedan orientar la labor del profesorado tutor y el aprendizaje autónomo del alumnado.

Finalmente, para facilitar el acceso y la localización de los recursos docentes de acción tutorial por parte de la comunidad universitaria y cumplir con los requisitos necesarios de publicación en acceso abierto, se recomienda: (1) integrar estos materiales en los repositorios institucionales de las universidades españolas y (2) gestionar una licencia de uso para garantizar el reconocimiento de su autoría.

\section{REFERENCIAS}

Álvarez, M. B., Lorca, P. \& García, J. (2010). El Programa de Acción Tutorial como complemento de la acción docente en el Espacio Europeo de Educación Superior (EEES). Revista de Educación en Contabilidad, Finanzas y Administración de Empresas, 1(1). Recuperado el 06 de julio de 2011, de www.educade.es/docs/01/02-alvarez.pdf

Cano, R. (2008). Modelo organizativo para la planificación y desarrollo de la tutoría universitaria en el marco del proceso de convergencia europea en Educación Superior. Revista Interuniversitaria de Formación del Profesorado, 22(1). Recuperado el 07 de julio de 2011, de dialnet.unirioja.es/servlet/articulo?codigo $=2541045$

Carrillo, V. \& Castillo, A. (2010). Responsabilidad social corporativa de la Universidad española en el entorno online. Trabajo presentado al Congreso AEIC Málaga. Recuperado el 15 de julio de 2011, de www.aeic.org/malaga2010/upload/ok/96.pdf

Casals, J. (2010). La contribución del Centro de Recursos para el Aprendizaje y la Investigación a la promoción del acceso abierto en la Universidad de Barcelona. Recuperado el 06 de junio de 2011, de openaccess.uoc.edu/webapns/o2/handle/10609/1733 
Del Valle, Á. (2004). La tutoría integrada en la vida ordinaria del alumno: espacios y niveles de actuación. Contextos educativos y acción tutorial. Madrid: Ministerio de Educación y Ciencia, Subdirección General de Información y Publicaciones.

Gairín, J., Feixas, M., Guillamón, C. \& Quinquer, D. (2004). La tutoría académica en el escenario europeo de la Educación Superior. Revista Interuniversitaria de Formación del Profesorado, 18(1). Recuperado el 30 de junio de 2011, de aufop.com/aufop/uploaded_files/articulos/1219255665.pdf

García, N. (2008). La función tutorial de la Universidad en el actual contexto de la Educación Superior. Revista Interuniversitaria de Formación del Profesorado, 22(1). Recuperado el 1 de julio de 2011, de redalyc.uaemex.mx/src/inicio/ArtPdfRed.jsp?iCve=27413170002

Hedlund, T. \& Rabow, I. (2007). Open Access in the Nordic countries: a state of the art report. Recuperado el el 1 de julio de 2011, de www.heal link.gr/SELL/OA_reports/NordicCountries.pdf

INFORME BRICALL. (2000). Universidad 2000. Madrid: Conferencia de Rectores de las Universidades Españolas (CRUE).

Jiménez, J. (2010). Hacia un nuevo proyecto de tutoría universitaria en el Espacio Europeo de Educación Superior. REOP, 21. Recuperado el 1 de julio de 2011, de www.uned.es/reop/pdfs/2010/21-1\%20-\%20Jorge\%20Jimenez.pdf

Labastida, I. \& Iglesias, C. (2006). Guía sobre gestión de derechos de autor y acceso abierto en bibliotecas, servicios de documentación y archivos. Recuperado el 3 de septiembre de 2011, de $\underline{\text { www.sedic.es/dchos_autor_normaweb.01.07.pdf }}$

Lázaro, Á. J. (2008). Diferencias cualitativas entre experiencias tutoriales para opciones de aprendizaje universitario. Revista Interuniversitaria de Formación del Profesorado, 22(1). Recuperado el 1 de julio de 2011, de redalyc.uaemex.mx/src/inicio/ArtPdfRed.jsp?iCve=27413170006

López, N. \& Sola, T. (2003). Orientación escolar y tutoría para las diferentes etapas de la educación. Granada: Grupo Editorial Universitario.

Llorens, F., Bayona, J.J., Gómez, J. \& Sanguino, F. (2010). The University of Alicante's institutional strategy to promote the open dissemination of knowledge. Online Information Review, 34 (4): 565-582. 
Lloret, A., Calatrava, E. \& Torres, M. J. (2010). Iniciativas de promoción del acceso abierto en RiuNet, Repositorio Institucional de la Universidad Politécnica de Valencia. Recuperado el 06 de junio de 2011, de openaccess.uoc.edu/webapps/o2/handle/10609/1710

Martínez, J. A. (2011). La orientación y la tutoría en el Espacio Europeo de Educación Superior. Cuadernos de Educación y Desarrollo, 23(3). Recuperado el 28 de junio de 2011 www.eumed.net/rev/ced/23/jamg.htm

Mateos, V. \& Montanero, M. (2008). Diseño e implementación de Títulos de Grado en el EEES. Madrid: Narcea.

Merlo-Vega, J.A. \& Ferreras, T. (2010). Políticas de promoción del acceso abierto en la Universidad de Salamanca. Recuperado el 06 de octubre de 2011, de openaccess.uoc.edu/webapps/o2/handle/10609/1729

Pantoja, A. \& Campoy, T.J. (2009). Planes de Acción Tutorial en la Universidad. Jaén: Universidad de Jaén, Servicio de Publicaciones e Intercambio.

Sáenz De Miera, A. (2001). En torno al trabajo universitario. Reflexiones y datos. Madrid: Secretaría General del Consejo de Universidades del MECD.

Sicinski, A. (1990). Objetivos educativos y valores culturales. En UNESCO: Sobre el futuro de la educación. Hacia el año 2000. Madrid: Narcea.

Sobrado, L. (2008). Plan de acción tutorial en los centros docentes universitarios: el rol del profesor tutor. Revista Interuniversitaria de Formación del Profesorado, 22(1). Recuperado el 30 de junio de 2011, de $\underline{\text { redalyc.uaemex.mx/src/inicio/ArtPdfRed.jsp?iCve=27413170005 }}$

Tortosa, M. T., Moliner, M., López, R. \& López, A. (2010). La orientación y el asesoramiento al alumnado que accede a la universidad: análisis de la accesibilidad al plan de acción tutorial. En Nuevas titulaciones y cambio universitario. Alicante: Universidad de Alicante.

Ley 14/1970, de 4 de agosto, General de Educación y Financiamiento de la Reforma Educativa.

Ley Orgánica 1/1990, de 3 de octubre, de Ordenación General del Sistema Educativo.

Ley Orgánica 2/2006, de 3 de mayo, de Educación.

Ley Orgánica 6/2001, de 21 de diciembre, de Universidades. 
Ley Orgánica 4/2007, de 12 de abril, por la que se modifica la Ley Orgánica 6/2001, de 21 de diciembre, de Universidades.

Real Decreto 1791/2010, de 30 de diciembre, por el que se aprueba el Estatuto del Estudiante Universitario.

\begin{abstract}
Alejandra Hernández Ruiz
Doctora en Comunicación Audiovisual y Publicidad. Profesora de la Universidad Complutense de Madrid, donde imparte clases en las áreas de Tecnología de los Medios Audiovisuales, Realización Audiovisual y Empresa Audiovisual. Miembro investigador de FONTA (Formación en Nuevas Tecnologías del Audiovisual). Especialista en Televisión Digital y Nuevas Tecnologías de la Sociedad de la Información. Compagina la actividad docente y de investigación con el trabajo en cadenas de televisión y productoras audiovisuales. Actualmente es Directora de Explotación de $13 \mathrm{TV}$.
\end{abstract}

\title{
Conchi Campillo Alhama
}

Profesora colaboradora del Dpto. de Comunicación y Psicología Social de la Universidad de Alicante. Licenciada en CC. de la Información (Publicidad y Relaciones Públicas) por la U.C.M. Doctora en Sociología por la Universidad de Alicante. Premio BLAS INFANTE 2010, de Estudio e Investigación sobre Administración y Gestión Pública, concedido por el Instituto Andaluz de Administración Pública (IAAP). Experta en Protocolo y RR. Institucionales por la Universidad Miguel Hernández y la Escuela Internacional de Protocolo (EIP). Ha desempeñado su actividad profesional en varias organizaciones. Pertenece al "Grupo de investigación de estudios de publicidad institucional, política y social" y a "Comunicación y públicos específicos".

\section{José Daniel Álvarez Teruel}

Doctor en Pedagogía por la Universidad de Murcia. Profesor Contratado Doctor en el Dpto. de Psicología Evolutiva y Didáctica de la U. de Alicante, del que ha sido Secretario. Imparte docencia en Magisterio, Psicopedagogía y en el programa de Doctorado "Diseño, Orientación e Intervención Psicopedagógica”. En la actualidad es Coordinador de Formación Docente y Coordinador del Programa de Acción Tutorial del Instituto de Ciencias de la Educación (ICE) de la U. de Alicante. Su línea de investigación y publicaciones giran en torno a la Acción Tutorial, la Evaluación Formativa y la Innovación Curricular. 